\title{
Fabrication of epitaxial nanostructured ferroelectrics and investigation of their domain structures
}

\author{
H. Han $\cdot$ K. Lee $\cdot$ W. Lee $\cdot$ M. Alexe $\cdot$ D. Hesse $\cdot$ \\ S. Baik
}

Received: 23 March 2009/Accepted: 27 April 2009/Published online: 16 May 2009

(c) The Author(s) 2009. This article is published with open access at Springerlink.com

\begin{abstract}
Nanostructured ferroelectrics are important objects for studies on ferroelectric size effects as well as for applications to memory devices with ultra-high memory density. In the present article, we introduce several approaches for the synthesis of confined ferroelectrics with sizes in and below the hundreds of nanometer range, including top-down processes like e-beam lithography, self-assembly methods like chemical solution deposition, and growth by pulsed laser deposition using stencil masks. Furthermore, the ferroelectric domain structure of part of these nanostructures is investigated by means of synchrotron X-ray diffraction, and its contribution to the ferroelectric properties is discussed.
\end{abstract}

\section{Introduction}

Ferroelectric materials have been and are still investigated intensively due to their wide range of electronic device applications making use of the exceptional physical properties, for example, dynamic random access memories (DRAMs), nonvolatile ferroelectric random access

H. Han $\cdot$ K. Lee $\cdot$ S. Baik $(\bowtie)$

Department of Materials Science and Engineering, Pohang

University of Science and Technology, Hyoja-dong,

Pohang 790-784, Korea

e-mail: sgbaik@postech.ac.kr

W. Lee

Korea Research Institute of Standards and Science,

Daejon 305-340, Korea

M. Alexe - D. Hesse

Max Planck Institute of Microstructure Physics, Weinberg 2, Halle 06120, Germany memories (NV-FERAMs) based on ferroelectric materials with high permittivity and polarization, respectively [1-3]. Requirements for the increase of data storage density and the decrease of production costs have driven the miniaturization of ferroelectrics as small as they can preserve the ferroelectricity [4-8]. For example, in order to achieve a memory density of $1 \mathrm{~Tb} / \mathrm{inch}^{2}$, one has to realize ferroelectric bits with sizes of less than $25 \times 25 \mathrm{~nm}^{2}$. Accordingly, intense efforts for the fabrication of nanostructured ferroelectrics have been contributed by many researchers to understand the ferroelectric size effects in low-dimensional nanostructures, in which ferroelectricity could be altered or even totally vanish at a certain critical size. The fabrication of lowdimensional ferroelectrics and their characterization, for example, ultra-thin films [9-14], nanotubes [15, 16], and nanowires [17-19], have well developed during last decades. Promising theoretical predictions as well as many interesting experimental observations of ferroelectricity by means of scanning force microscopy and synchrotron X-ray diffraction have been reported $[9,11,20,21]$. These observations confirmed that ferroelectricity could be preserved in films of very low thickness due to the reduction of the depolarization energy by forming nanoscale $180^{\circ}$ stripe domain patterns on insulating substrates or due to the presence of conducting metallic electrodes, resulting in a stable polarization state at a thickness of several unit cells. In a theoretical work it was reported that the interface between an ultra-thin ferroelectric thin film and a paraelectric substrate could affect the ferroelectric properties due to correlations of atomic displacements through the interface [22]. In real systems, however, structural defects such as interfacial dislocations [23, 24] or imperfect screening by conducting electrodes [10] may affect the ferroelectric properties. Overall one can say that the fundamental limit of thickness in ferroelectric films has been overcome. 
Contrary to the thickness limit of ferroelectricity, the size effect resulting from a lateral size limit, i.e., the question how small a ferroelectric nanostructure can become without loosing the ferroelectric properties, is still unveiled due to difficulties in the fabrication of uniform and reproducible ferroelectric nanostructures of very small sizes. There were several theoretical studies on the size effect showing that ferroelectric and piezoelectric properties could be altered due to the increased influence of the surfaces and to reduced substrate constraints [6, 25-27]. Recently, experimental [28-31] and theoretical [32] observations suggested that confined ferroelectrics could have unique properties. Nagarajan et al. [28] showed that the ferroelectric and piezoelectric properties were increased in discrete ferroelectric films owing to a reduced substrate clamping effect. Lee et al. prepared PZT patterns with morphotropic phase boundary (MPB) composition utilizing the strain relaxation in ferroelectric islands [29]. Bühlmann et al. [30] explained that the increase of piezoresponse in nanoislands originated from a reduced share of $a$-domains. Naumov et al. [32] found by ab initio studies that the ferroelectrics could have toroidal polarization states in zero-dimensional ferroelectric particles with a size of $3.2 \mathrm{~nm}$, which would principally enable a memory density of $60 \mathrm{~Tb} / \mathrm{inch}^{2}$.

Various experimental approaches to obtain nanostructured ferroelectrics with small size, good ordering, and high quality have been recently developed, including so called top-down [33-37] and bottom-up methods [38-42]. Top-down methods, for example, e-beam direct writing (EBDW), e-beam patterning, focused ion-beam (FIB) milling, and nano imprint lithography are useful approaches in view of precise size control and ordering of nanofeatures. However, the large-scale operation of these methods is expensive, time-consuming and above all, the ferroelectric properties are frequently affected by mechanical damage or e-beam or ion-beam damage of the surface-near areas of the nanostructures [34, 43]. On the other hand, bottom-up methods such as metal-organic vapor deposition (MOCVD), chemical solution deposition (CSD), and hydrothermal methods may enable the preparation of even smaller ferroelectric nanostructures and to study intrinsic size effects. Roelofs et al. [41] showed that no piezoresponse was detected in $\mathrm{PbTiO}_{3}$ nanograins with sizes below $20 \mathrm{~nm}$, which can be the size limit where ferroelectricity can still exist. Shimizu et al. observed that $\mathrm{PbTiO}_{3}$ nanoislands with $38 \mathrm{~nm}$ lateral size still show a piezoresponse when studied by atomic force microscopy [44]. Although bottom-up methods permit the investigation of extremely miniaturized regions of ferroelectrics, they have other limitations. Not only are there difficulties in precisely locating each bit (each nanostructure) in a wellordered array, but also in controlling the size of the latter exactly. For taking advantages of both top-down and bottom-up approaches, selective growth using various mask or seed patterns is being investigated, for example, nanosphere- [45], metal nanotube membrane- [46, 47], and template-based growth [48, 49]. Due to the uniform size and well-ordered arrays of ferroelectric nanostructures in these cases, the authors were able to measure the structural orientation by laboratory-based XRD, and determine their piezoelectric properties [47]. Most recently, novel techniques to synthesize unique structures of ferroelectrics have been employed. Zhu et al. [50] showed that using AAO templates could be the way to realize 5-nm sized ferroelectric ring structures which have a potential to demonstrate the vortex polarization suggested by Naumov. Evans et al. [51] fabricated self-assembled nanocapacitors with nanosized Pt electrodes by means of AAO.

Despite the multifarious activities for studies of nanostructured ferroelectrics, there have been no detailed reports which would give a clear understanding of domain structures in ferroelectric nanoislands. Since the ferroelectric properties and device performances are a function of the crystallographic orientations and the domain structures in ferroelectric thin films, a wide range of work has been devoted to understand the formation mechanisms of domains, the role of domain boundaries, and the controlling factors of the domain structure [52-54]. Jesse et al. [55] revealed by switching spectroscopy PFM (SS-PFM) at the nanometer scale that $90^{\circ}$ domain boundaries can be nucleation centers for $180^{\circ}$ polarization switching. Jia et al. [56] found in atomic-scale high-resolution TEM investigations of PZT films that charged $180^{\circ}$ domain boundaries with head-to-head or tail-to-tail configuration of the electric dipoles are about 10 times broader than non-charged $180^{\circ}$ boundaries. Thus, one may assume that the charged domain boundaries are more mobile than the uncharged ones, so that the $180^{\circ}$ domains quickly grow in the polarization direction rather than showing lateral growth. However, more understanding of domain structures and their effects onto ferroelectric and piezoelectric properties in nanosized ferroelectrics is desirable, because the domain structures can be significantly changed by the different strain state in nanostructures compared to bulk or thin film ferroelectrics.

In this article, we discuss recent results of our work, mainly focusing on the fabrication methods of nanostructured ferroelectrics by e-beam lithography, self-assembly, laser interference lithography and template masks, as well as investigations of domain structures and their effects on the electrical properties. The domain structure was investigated by reciprocal space mapping techniques with synchrotron XRD, and the electrical properties were measured by $\mathrm{AFM}$ in the piezoresponse mode. 


\section{Experimental procedure}

Fabrication methods for nanostructured ferroelectrics

\section{e-beam lithography}

Electron-beam lithography was performed in a scanning electron microscopy (SEM) system (JEOL, Japan) equipped with a beam blanker (Deben, UK) controlled by the Elphy Plus software (RAITH, Germany). MMA-MAA EL6 (Microchem, USA) films with a thickness of about $150 \mathrm{~nm}$ were used as e-beam sensitive resist. For patterning, an accelerating voltage of $29 \mathrm{kV}$, an e-beam spot size of $10 \mathrm{~nm}$, and an area dose of $200 \mu \mathrm{C} / \mathrm{cm}^{2}$ were used. Platinum with a thickness of $100 \mathrm{~nm}$ was deposited by e-beam evaporation to both serve as etching mask and top electrode after developing the e-beam pattern. Then, rinsing in acetone for lift-off and in methanol, followed by deionized water was carried out. An inductively coupled plasma (ICP) advanced oxide etcher (AOE) (STS, UK) was used for the etching process. The Pt top electrode was etched by $\mathrm{Ar}$ and $\mathrm{He}$ with a flow of 15 and $10 \mathrm{sccm}$, respectively. Then, $\mathrm{C}_{4} \mathrm{H}_{4}, \mathrm{C}_{2} \mathrm{H}_{2}$, Ar, and $\mathrm{O}_{2}$ with $5,10,5$, and $10 \mathrm{sccm}$, were used for the etching of 100 -nm-thick $\mathrm{PbTiO}_{3}$ patterns. Patterned samples were annealed in oxygen ambient at $600{ }^{\circ} \mathrm{C}$ for $1 \mathrm{~h}$ to remove any etching-related damages.

\section{Chemical solution deposition (CSD)}

For CSD, commercial $\mathrm{PbTiO}_{3}$ polymeric precursors with a concentration of $0.3 \mathrm{~mol} / \mathrm{L}$ and $10 \%$ excess $\mathrm{PbO}$ were dissolved in 2-methoxyethanol. The initial thickness was varied by varying the dilutions of the raw precursor in the range of 1:5 to $1: 120 . \mathrm{PbTiO}_{3}$ solutions were spin-coated on selected substrates at $5000 \mathrm{rpm}$ for $30 \mathrm{~s}$, and the obtained ultra-thin gel film was kept in air for at least $30 \mathrm{~min}$. As-deposited films were pyrolized at $450{ }^{\circ} \mathrm{C}$ for $1 \mathrm{~h}$ in a box furnace; then the specimens were heated to high temperatures, for example, to $650-800{ }^{\circ} \mathrm{C}$, and kept for $1 \mathrm{~h}$ to crystallize the pyrolized film. The sizes of the nanoislands were controlled by the initial thickness of the as-deposited film, by the annealing temperature and the annealing time.

\section{Ultra-thin anodic alumina mask (UTAAM)}

Self-ordered UTAAM with uniform channels could be synthesized by two-step anodization of surface-finished pure aluminum plates [57, 58]. Initially, the aluminum plate was electropolished in a mixture of $\mathrm{HClO}_{4}$ and $\mathrm{C}_{2} \mathrm{H}_{5} \mathrm{OH}=1: 3$, then the first anodization was carried out in electrolytes under a constant cell potential for $12 \mathrm{~h}$.
After the first anodization, the anodic alumina was completely etched in chromic acid (mixture of $\mathrm{H}_{2} \mathrm{CrO}_{4}, \mathrm{H}_{3} \mathrm{PO}_{4}$, and deionized water). By making use of self-ordered structural arrays of pores on the aluminum surface, porous anodic alumina with uniform channels could be formed after the second anodization. For the use of porous alumina as a hard mask, the thickness of AAO was strictly controlled. For example, the thickness of the AAO after oxalic acid anodization was $400 \mathrm{~nm}$ in order to obtain an aspect ratio of about 6 , which is sufficient for PZT deposition through the pores. After the AAO membrane was synthesized, the remaining aluminum and the barrier oxide layer at the pore bottom were removed. The key step for the fabrication of the UTAAM is polystyrene (PS) coating. The PS coating layer protects the ultra-thin hard mask from cracking during the etching step of the aluminum and the barrier layer. A solution of PS in $\mathrm{CHCl}_{3}$ was spin-coated at $3000 \mathrm{rpm}$ for $30 \mathrm{~s}$, and then the solvent was evaporated at $80^{\circ} \mathrm{C}$. The rest of aluminum was etched in a mixed solution of $\mathrm{CuCl}_{2} \cdot 2 \mathrm{H}_{2} \mathrm{O}$, deionized water, and $\mathrm{HCl}$, followed by $\mathrm{HNO}_{3}$-rinsing for the complete removal of the residual copper on the surface of the mask. Barrier layers of AAO prepared in phosphoric, oxalic, and sulfuric acid were removed by $10 \mathrm{wt} \%$ of $\mathrm{H}_{3} \mathrm{PO}_{4}$ at $45{ }^{\circ} \mathrm{C}$ for $60 \mathrm{~min}, 5 \mathrm{wt} \%$ of $\mathrm{H}_{3} \mathrm{PO}_{4}$ at $30{ }^{\circ} \mathrm{C}$ for $30 \mathrm{~min}$ and $5 \mathrm{wt} \%$ of $\mathrm{H}_{3} \mathrm{PO}_{4}$ at $30{ }^{\circ} \mathrm{C}$ for $15 \mathrm{~min}$, respectively. During the etching of the barrier layer, the pore walls were protected from the etching solution due to the PS in the pores. The PS layer makes the UTAAM float on the deionized water and on the $\mathrm{H}_{3} \mathrm{PO}_{4}$ solution due to its hydrophobic nature. The mask was taken out of the deionized water, floating it directly onto the substrate for deposition, and the PS was removed by $\mathrm{CHCl}_{3}$ or oxygen plasma etching, resulting in a hard mask for deposition.

\section{Laser interference lithography (LIL)}

First, an antireflection coating (ARC, XHRiC-11, Brewer Science, US) layer with a thickness of $150 \mathrm{~nm}$ was spincoated at $2500 \mathrm{rpm}$ and baked at $180{ }^{\circ} \mathrm{C}$ for $60 \mathrm{~s}$. Then a $\mathrm{SiO}_{2}$ mask layer was deposited on the ARC by sputtering. A $180 \mathrm{~nm}$ thick negative photoresist (PR, TSMR-iN027, OHKA, Japan) was spin-coated at $600 \mathrm{rpm}$ and pre-baked at $90{ }^{\circ} \mathrm{C}$ for $90 \mathrm{~s}$. The PR was post-baked at $110{ }^{\circ} \mathrm{C}$ for $90 \mathrm{~s}$ after laser exposure. For interference lithography, a $\mathrm{HeCd}$ laser with a wavelength of $325 \mathrm{~nm}$ and a Lloyd's mirror interferometer were used. Two coherent beams generate periodic interference patterns on the PR, and the patterns were transferred to the $\mathrm{SiO}_{2}$ mask layer by reactive ion etching (RIE). $\mathrm{CHF}_{3}$ gas was used to open the $\mathrm{SiO}_{2}$ layer and the subjacent ARC layer during the RIE step. The residual $\mathrm{PR}$ on top of the $\mathrm{SiO}_{2}$ layer was also removed by $\mathrm{O}_{2}$ plasma under operating conditions of $10 \mathrm{mTorr}$ and 
$100 \mathrm{~W}$ in the RIE step. The lift-off process of the ARC layer was carried out by a RCA-1 ( $\mathrm{H}_{2} \mathrm{O}: \mathrm{NH}_{4} \mathrm{OH}$ : $\left.\mathrm{H}_{2} \mathrm{O}_{2}=5: 1: 1\right)$ cleaning solution at $70{ }^{\circ} \mathrm{C}$ for $1 \mathrm{~h}$ in the lift-off step after pulsed laser deposition. By this lift-off of the ARC layer, the $\mathrm{SiO}_{2}$ mask was simultaneously taken away from the substrate.

\section{Pulsed laser deposition (PLD)}

The ferroelectric material was deposited onto the substrates by PLD with a $\operatorname{KrF}$ eximer laser $(\lambda=248 \mathrm{~nm})$ at an energy fluence of $400-600 \mathrm{~mJ} / \mathrm{cm}^{2}$. The background pressure was below $1 \times 10^{-6}$ Torr to give a high energy to the particles emanating from the target, so that they can reach the substrate surface through the $\mathrm{SiO}_{2}$ and $\mathrm{AAO}$ masks.

\section{Structural characterizations}

\section{High-resolution synchrotron $X$-ray diffraction (XRD)}

The domain structure of ferroelectric nanoislands was observed by high-resolution synchrotron XRD installed on the Huber six-circle diffractometer of $3 \mathrm{C} 2$ and $10 \mathrm{C} 1$ beam lines at the Pohang Light Source (PLS). The incident beam size was less than $1 \mathrm{~mm}^{2}$ and the energy was $8 \mathrm{keV}$. Reciprocal space mapping was used for the investigation of the final domain structure in the ferroelectric nanoislands.

\section{Transmission electron microscopy (TEM)}

Conventional TEM investigations were carried out in a Philips CM 20 Twin (Philips, Netherlands) microscope, whereas HRTEM investigations were performed in a Jeol 4010 (Jeol, Japan) microscope. Some of the thin samples for TEM investigations were prepared by standard methods of mechanical polishing and ion milling. The other samples were prepared by FIB milling using a gallium ion beam in an FEI Nova 600 NanoLab system.

\section{Functional characterization}

\section{Piezoresponse force microscopy (PFM)}

Finally, PFM was employed for the measurement of the electrical properties, such as switching behavior, and piezoresponse of each nanoisland under an external electric field. A CP-Research atomic force microscope was used in contact mode to record the piezoelectric signals from the nanoislands, using a conducting AFM tip with an elastic constant of $2.5 \mathrm{Nm}$ and a lock-in amplifier.

\section{Results and discussions}

Non-tilted $a$-domains in nanostructured ferroelectrics

The final domain structure in ferroelectric thin films epitaxially grown on a suitable substrate is governed by misfit strain due to the lattice mismatch between the film and the substrate at growth temperature, and by transformation strain below the Curie temperature, followed by their relaxation via dislocation generation at the interface and via polydomain formation, respectively [59, 60]. In tetragonal ferroelectrics where $c$ - and $a$-domains can coexist, $180^{\circ}$ and $90^{\circ}$ domain walls are formed at the phase transformation near the Curie temperature, to reduce the depolarizing field and the elastic energy. Adjacent $a$ - and $c$-domains form coherent twin boundaries along $\{101\}$ planes which can lead to a unique periodic $c / a / c / a$ polydomain structure. In order to reveal the differences between the domain structures of a continuous film and a nanopattern, we fabricated an array of 734,400 discrete $\mathrm{PbTiO}_{3}$ dots with a size of $100 \mathrm{~nm}$ by e-beam lithography and carried out the reciprocal space mapping. Figure 1 shows contour maps of the $H L$ - and $H K$-planes near the $\mathrm{PbTiO}_{3}$ (001) position in reciprocal space, obtained by help of a synchrotron X-ray source, presenting the domain structures of $\mathrm{PbTiO}_{3}$, viz. (a)-(c) of a $150 \mathrm{~nm}$ thick film, and (d)-(f) of a $100 \mathrm{~nm}$ sized nanopattern grown on $\mathrm{Pt}$ (100)/MgO (100). In the measurement, the $\mathrm{MgO}$ substrate (cubic lattice parameter, $a=4.213 \AA$ ) was used as an internal standard for the reciprocal lattice, assuming that the substrate is unstrained. The reciprocal space mapping of the $H L$-plane was conducted by keeping the Miller index $k$ and varying $h$ by $\pm \Delta h$ every increment $\Delta l$ in $l$. The $\mathrm{PbTiO}_{3}(001)$ and (100) reflections in the $H L$-plane maps correspond to $c$ - and $a$-domains, respectively. In the continuous $\mathrm{PbTiO}_{3}$ film, symmetric $c$-domains and 4-fold symmetric $a$-domains due to the tilting angle of $90^{\circ}$ domain walls were observed in the $H K$-plane maps, which present the cross-sections of each $c$ - and $a$-domain region as shown in Fig. 1c. A new type of ferroelastic $90^{\circ}$ domain structure was observed in the center of a tilted $a$-domain region in the $H L$-plane of the reciprocal lattice map of $100 \mathrm{~nm}$ sized ferroelectric patterns, as shown in Fig. 1d. The $H K$-plane map of an $a$-domain in Fig. If clearly shows five peaksfour symmetric ones, and the central one, all corresponding to $a$-domains. This type of $a$-domain is aligned with its $c$-axis exactly parallel to the substrate plane, without the usual tilting angle which is required to maintain $c / a / c / a$ twinned polydomain structures. Figure 2 shows the illustrations of two types of $a$-domains, (a) the normal twin $a$-domains $(\Delta \omega \neq 0)$ and (b) the non-tilted $a$-domains $(\Delta \omega=0)$, where $\Delta \omega=90^{\circ}-2 \tan ^{-1}(a / c)$, the latter being newly found in the reciprocal space maps of $100 \mathrm{~nm}$ 

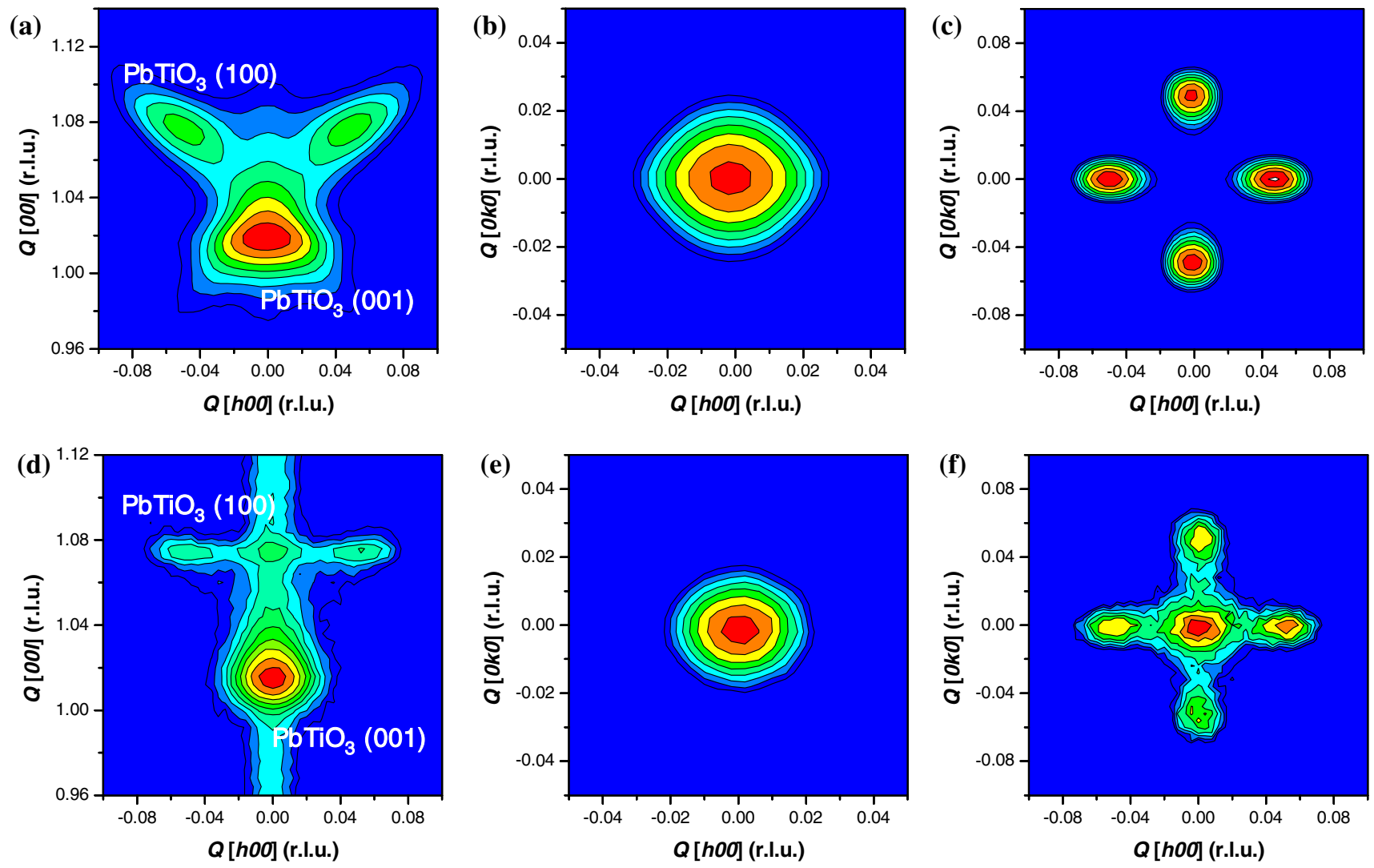

Fig. 1 Contour plots of reciprocal space mapping of epitaxial $\mathrm{PbTiO}_{3}$ a-c continuous film and d-f 100-nm sized nanopatterns obtained near $\mathrm{PbTiO}_{3}(001)$ and (100) peaks. a, d $H L$-plane scan, b, e $H K$-plane scan of $\mathrm{PbTiO}_{3}(001)$, and c, f $H K$-plane scan of $\mathrm{PbTiO}_{3}$ (100)

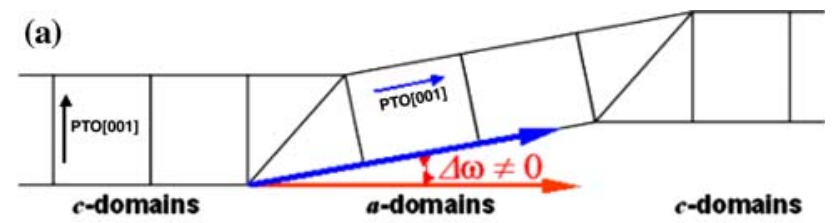

(b)

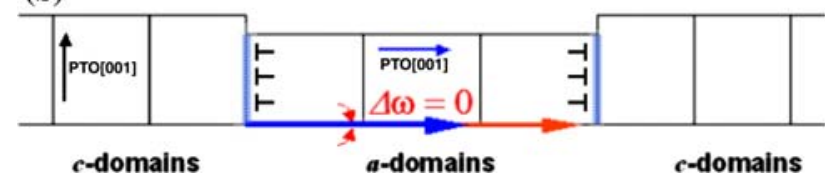

Fig. 2 Schematics illustrating two types of $a / c$ domains observed in $100 \mathrm{~nm}$ sized $\mathrm{PbTiO}_{3}$ nanopatterns; a with tilted $(\Delta \omega \neq 0)$ and $\mathbf{b}$ with non-tilted $a$-domains $(\Delta \omega=0)$. It should be noted that the structure shown in $\mathbf{b}$ is only a schematic in order to help understand the term "non-tilted $a$-domains" (From Ref. [58])

sized $\mathrm{PbTiO}_{3}$ nanopatterns. Here, it should be noted that the sketch of a non-tilted $a$-domain shown in Fig. $2 \mathrm{~b}$ is only a principle guide for understanding. In particular, the bounding ferroelectric domain walls should be uncharged from the point of energy minimization. Only under this condition the formation of non-tilted $a$-domains would be reflections, corresponding to $c$-domains and $a$-domains, respectively (Reprinted with permission from Ref. [62]. Copyright 2009, American Institute of Physics)

energetically favorable. The habit plane of $90^{\circ} \mathrm{a} / \mathrm{c}$ domain boundaries usually is (110) as shown in Fig. 2a, and 4-fold symmetric $a$-domains with $45^{\circ}$ (110) twin boundaries are normally present in epitaxial continuous ferroelectric films and even in patterns of larger ferroelectric islands such as ones of a diameter of several micron [61]. However, as the size of the islands decreases further and reaches $100 \mathrm{~nm}$, $a$-domains without tilting angle begin to be present [62]. Recently, Jia et al. [56] have shown that it is possible that charged domain boundaries exist due to the stabilization by aliovalent cations (i.e., $\mathrm{Ti}^{3+}$ in PZT).

When the lateral size of a ferroelectric nanostructure decreases while the thickness is kept constant, the degree of misfit strain relaxation increases. The misfit strain relaxation is strongly dependent on the scaling ratio and occurs predominantly in the edge region of discrete patterns. In the nanopatterns, the misfit strain at the edge regions of the patterns is fully relaxed [4], resulting in the appearance of non-tilted $a$-domains. We had initially assumed that single-domain structures would appear in nanosized ferroelectrics. However, in reality the $a$-domain abundance rather increases and the described new type of $a$-domains appears in nanosize islands. It remains to be 
explained how the new and the conventional $a$-domains are distributed in the small features. If the size of the ferroelectric islands decreases further, then there might be a critical size below which the periodic $c / a / c / a$ domain structures with $45^{\circ}$ twin boundaries are unfavorable. Therefore, one needs to prepare even smaller sized ferroelectrics and investigate their domain structures. However, it occurred to be difficult to fabricate ferroelectric nanostructures with a lateral size of only several tens of nanometers by a top-down method such as e-beam lithography. Thus, we employed a bottom-up approach, i.e., CSD, for the next step of the investigations.

Piezoresponse and $a$-domains in nanostructured ferroelectrics

$\mathrm{PbTiO}_{3}$ nanoislands with average size ranging from 450 to $55 \mathrm{~nm}$ were fabricated by CSD, simply controlled by dilution of solution, annealing time and temperature (H. Han et al., unpublished). Figure 3 shows representative AFM images of $\mathrm{PbTiO}_{3}$ nanoislands with average size $(x)$ and standard deviation $(\sigma)$ of (a) $52.2 \mathrm{~nm}, 45.8 \mathrm{~nm}$; (b) $102.3 \mathrm{~nm}, 67 \mathrm{~nm}$; (c) $223.7 \mathrm{~nm}, 99.3 \mathrm{~nm}$; and (d) $497 \mathrm{~nm}, 280.3 \mathrm{~nm}$, respectively. The insets in the AFM images show the size distributions obtained from each image by a home-built image analysis software. The various sizes in Fig. 3 were obtained by changing the dilution rates of the solutions while other parameters were kept constant. The scaling ratios $(f=$ lateral size/thickness) of all islands are between 3 and 6 which indicates that the lateral dimensions scale down with the thickness. For the comparison of the piezoresponse between ferroelectric nanostructures and continuous films, we also fabricated $\mathrm{PbTiO}_{3}$ thin films with a thickness of 10, 20, 40, and $80 \mathrm{~nm}$ by PLD. XRD analysis confirmed that all the $\mathrm{PbTiO}_{3}$ nanoislands were grown epitaxially on $\mathrm{Pt}(100) / \mathrm{MgO}$ (100). We measured the piezoresponse of nanoislands with sizes ranging from less than $50 \mathrm{~nm}$ to several hundreds of nanometers by probing all samples fabricated by different degrees of dilution. Figure 4 shows (a) topography and (b) piezoresponse image of nanoislands grown by CSD. In the as-grown state, the islands showed randomly poled states. Piezoresponse was detected even in less than $20 \mathrm{~nm}$ sized nanoislands, although some of them showed no signal
Fig. 3 AFM images of $\mathrm{PbTiO}_{3}$ nanoislands with average size $(x)$ and standard deviation $(\sigma)$ of a $52.2 \mathrm{~nm}, 45.8 \mathrm{~nm}$; b $102.3 \mathrm{~nm}, 67 \mathrm{~nm}$; $223.7 \mathrm{~nm}$, $99.3 \mathrm{~nm}$; and $\mathbf{d} 497 \mathrm{~nm}$, $280.3 \mathrm{~nm}$, respectively. Insets in each image are histograms of the size distribution obtained by an image analysis software applied to the AFM images
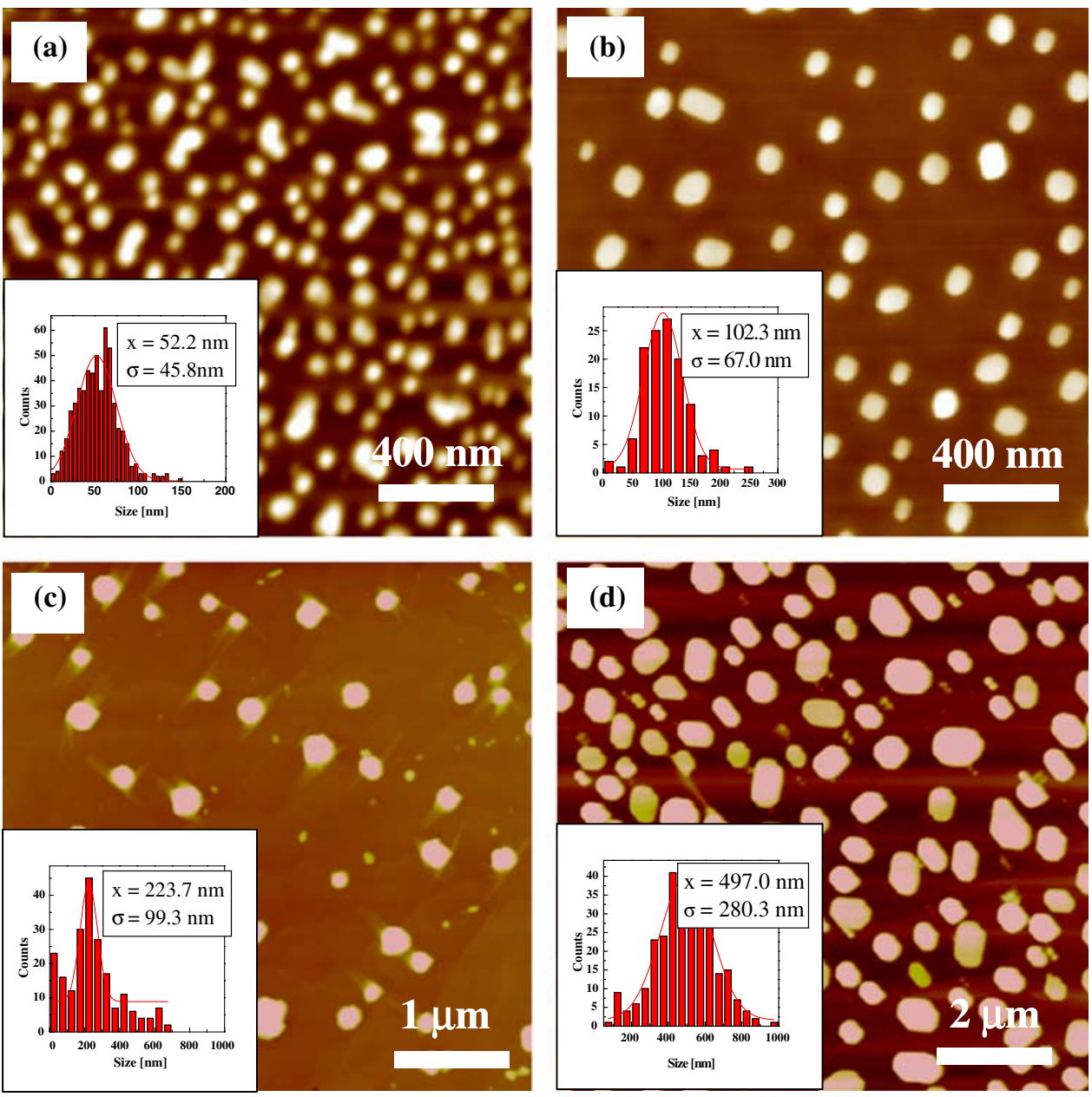

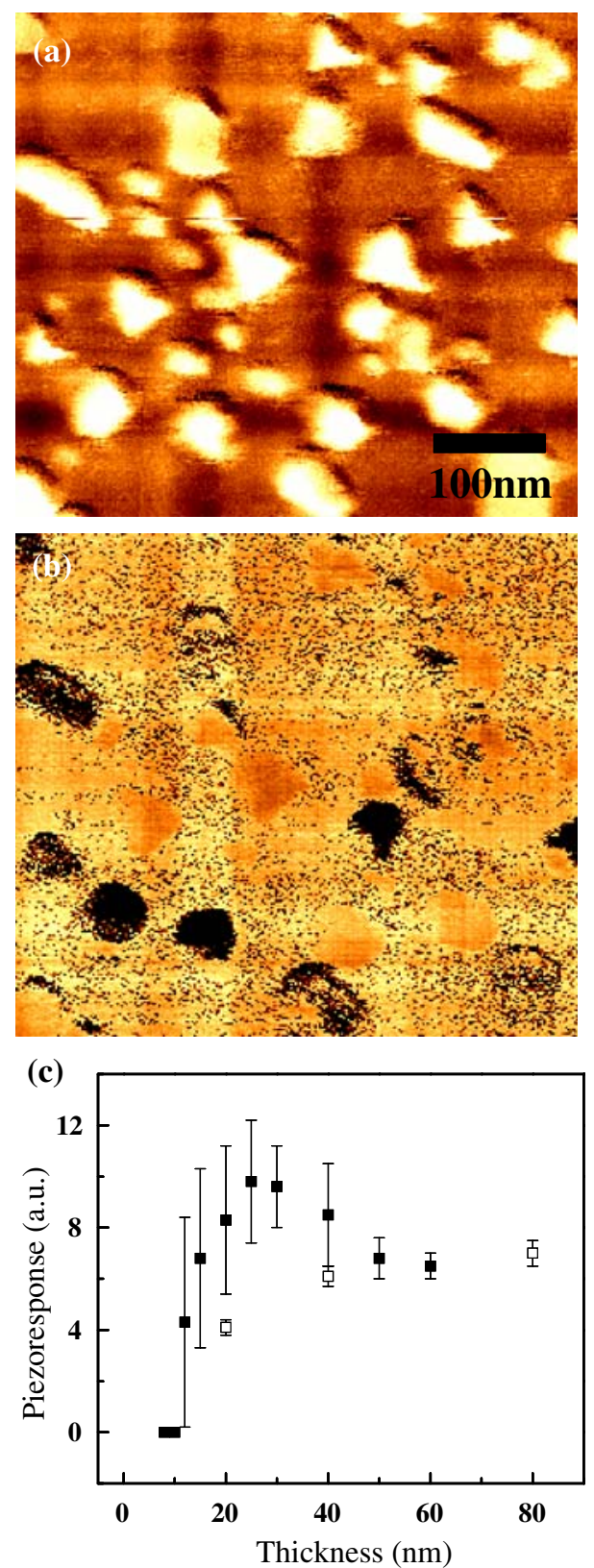

Fig. 4 PFM measurement. a Topography and $\mathbf{b}$ piezoresponse image obtained from $\mathrm{PbTiO}_{3}$ nanoislands. c Piezoresponse as a function of thickness of $\mathrm{PbTiO}_{3}$ nanoislands (solid squares) and thin films (open squares) measured by local hysteresis analysis on each nanoisland and film

as shown in Fig. 4b. Local hysteresis measurements were carried out in each island by applying an external electric field from -5 to $5 \mathrm{~V}$, and the amplitudes of switching curves were collected for the statistical analysis. From hysteresis measurement, we found that the coercive fields were independent from the size but the piezoresponse showed an interesting tendency shown in Fig. 4c. Even though the thickness of the nanoislands decreased, there was no reduction of the piezoelectric response, but even a slight increase at a thickness down to $25 \mathrm{~nm}$ (solid squares in Fig. 4c). When the thickness of the nanoislands was below $20 \mathrm{~nm}$, the piezoresponse signal converged to zero and finally no signal was detected at all in $10 \mathrm{~nm}$ thin islands. Piezoresponse as a function of the lateral size of the nanoislands showed a tendency similar to that of the thickness dependence. In contrast, a systematic reduction of the piezoresponse with the decrease in thickness was observed in continuous thin films (open squares in Fig. 4c). Thin films with a thickness of $80 \mathrm{~nm}$ showed a similar value of the piezoresponse as islands of smaller thickness. No piezoresponse was also detected in 10-nm-thick films. Bühlman and Nagarajan have shown that the piezoresponse of patterned ferroelectrics could increase [28, 30]. But the sizes they observed were in the range of several hundreds of nanometer and they explained the behavior in a different way. Nagarajan proved that the switching of $90^{\circ}$ domains can contribute to an increase of piezoresponse in patterned structures due to the significantly reduced substrate clamping [28]. Bühlman explained that vanishing $90^{\circ}$ domains mainly lead to an increase of piezoresponse [30].

We performed investigations of the domain structure in CSD-grown islands by reciprocal space mapping using high-resolution synchrotron XRD. Figure 5 shows reciprocal space maps of $H L$-plane mesh scans [(a)-(d)] and $H K$-plane mesh scans of $a$-domains [(e)-(h)] of $\mathrm{PbTiO}_{3}$ nanoislands with a mean lateral size and thickness of (a) $490 \mathrm{~nm}, 95 \mathrm{~nm}$; (b) $230 \mathrm{~nm}, 55 \mathrm{~nm}$; (c) $105 \mathrm{~nm}, 35 \mathrm{~nm}$; (d) $52 \mathrm{~nm}, 13 \mathrm{~nm}$, respectively. The $H L$-plane mesh scan of the islands with the lateral size and thickness of 490 and $95 \mathrm{~nm}$ and the $H K$-plane mesh of $a$-domains show distinctly separated $c$-domains, 4 -fold symmetric $a$-domains and a very small amount of non-tilted $a$-domains. As the size of the islands decreases further, however, it appears that the peaks of the $a$-domain regions become closer to that of the $c$-domain region. In the case of nanoislands with a lateral size of $52 \mathrm{~nm}$ and a thickness of $13 \mathrm{~nm}$, no $a$-domain peaks with off angle from the substrate normal are observed in the contour map of the $H L$-plane. The $H K$-plane mesh scans clearly show that with decreasing size the $a$-domain structures convert from 4-fold symmetric twinned $a$-domains to one symmetric peak. Unlike the center peak from the non-tilted $a$-domain in the $H K$-plane mesh of Fig. 1f, it is assumed that the center peaks in Fig. 5e-h came from both the shoulder of the $c$-domain and the 4 -fold symmetric $a$-domains as well as the simple peak broadening of XRD intensity along the orthogonal direction due to the very thin layer structure as clearly seen in the $H L$-mesh of Fig. $5 \mathrm{a}-\mathrm{d}$.

The exact amounts of $a$ - and $c$-domains were obtained by rocking scans along the $\chi$-direction while the angles $\omega$ and $2 \theta$ were fixed. This was performed for $2 \theta$ values 

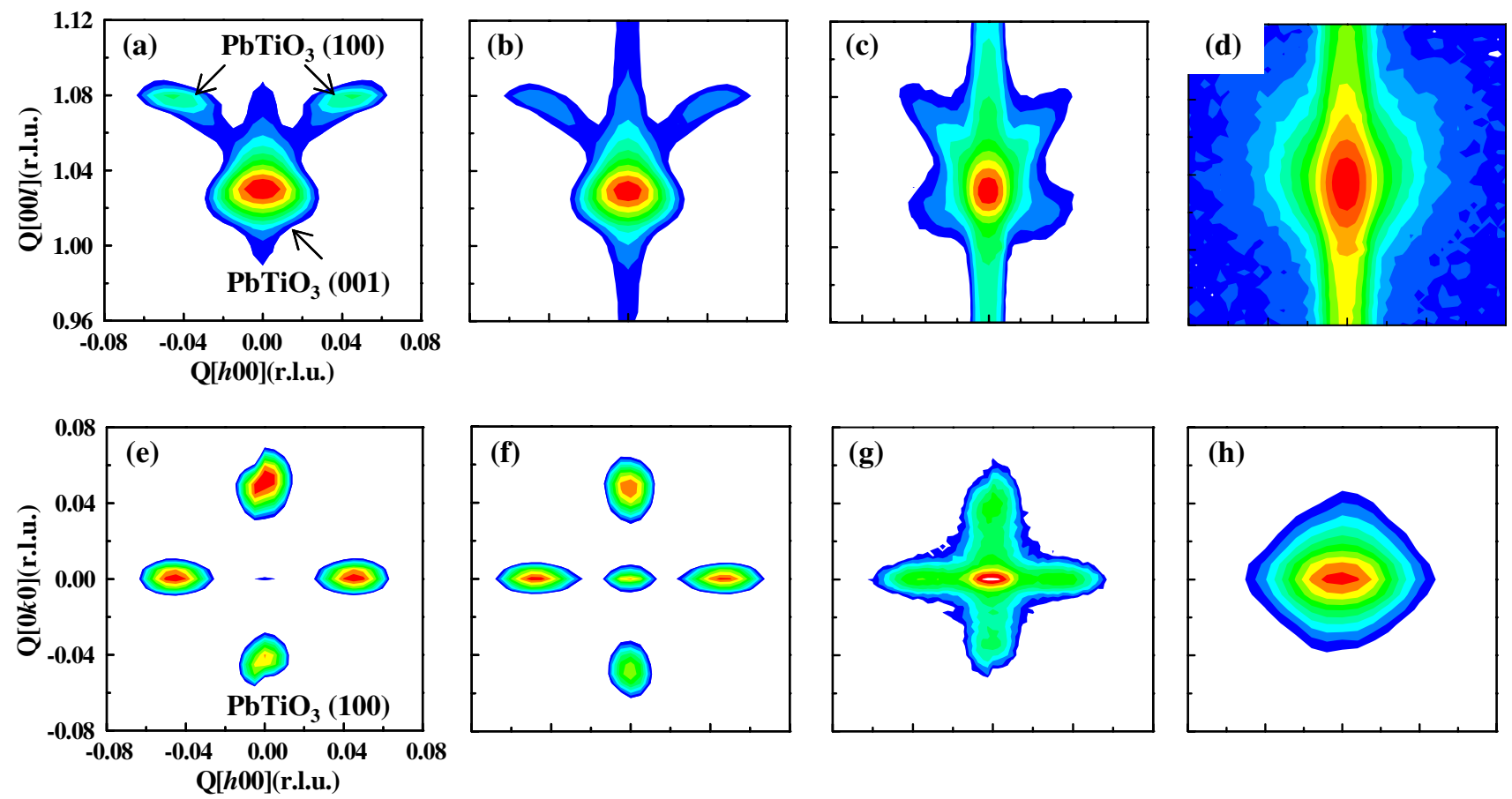

Fig. 5 Contour plots of reciprocal space mapping of a-d $H L$-plane scan near $\mathrm{PbTiO}_{3}(001)$ and (100) reflections and $\mathbf{e}-\mathbf{h} H K$-plane scan of $\mathrm{PbTiO}_{3}$ (100) reflections of $\mathrm{PbTiO}_{3}$ nanoislands with average size of $\mathbf{a}, \mathbf{e} 490 \mathrm{~nm} ; \mathbf{b}, \mathbf{f} 230 \mathrm{~nm} ; \mathbf{c}, \mathbf{g ~} 105 \mathrm{~nm}$; and $\mathbf{d}, \mathbf{h} 52 \mathrm{~nm}$, respectively

Fig. 6 a $c$-domain abundance as a function of thickness of $\mathrm{PbTiO}_{3}$ nanoislands (solid squares) and thin films (open squares), b Dependence of the abundances of non-tilted $a$ domains (open circles) and twinned $a$-domains (solid circles) as a function of the lateral size of $\mathrm{PbTiO}_{3}$ nanoislands

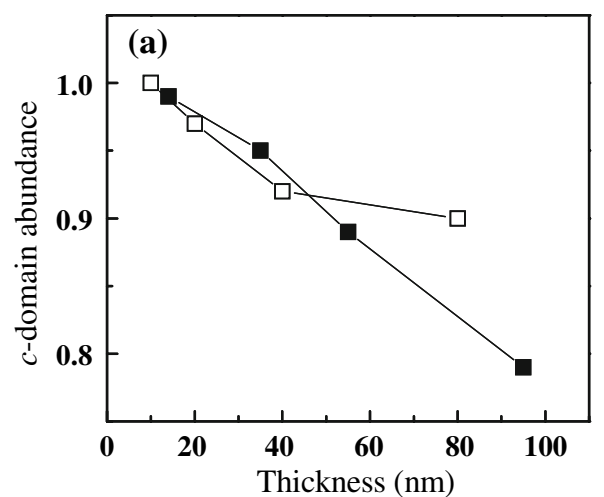

corresponding to the Bragg angle (001) and (100), respectively, calculating the intensity of each of the domains, e.g., $\alpha=\Sigma I(001) / \Sigma\{I(001)+I(100)\}$ for the $c$-domain abundance. Figure 6 a shows the $c$-domain abundance as a function of the thickness of $\mathrm{PbTiO}_{3}$ nanoislands (solid squares) and continuous films (open squares). As the thickness of both nanoislands and thin films decreases, the degree of $c$-axis orientation increases gradually. As the size of nanoislands and thin film decreased, the periodic $c / a / c / a$ domain structures with twin boundaries became unfavorable, and single $c$-domain structures became stable. In thin films, it is known that $90^{\circ}$ domains play a role in reducing the residual misfit strain and also influence the electrical properties. Kim et al. [31] have shown that the piezoelectric coefficient increased as the thickness of epitaxial $\mathrm{PbTiO}_{3}$ films on $\mathrm{Pt}$ bottom electrode decreased down from 200 to $60 \mathrm{~nm}$ due to the reduced $a$-domains. As the thickness of the film decreases further to $10 \mathrm{~nm}$, the amount of $90^{\circ}$ domains decreases and the films undergo a strong substrate clamping and domain pinning by depolarization field [10]. This can be one of the reasons for the decrease of the piezoresponse at decreasing thickness, despite the high tetragonality and reduction of $a$-domains in thin films. In the nanoislands, $a$-domains decreased with decreasing scales and the tetragonality is smaller than that of thin films. Therefore, it is supposed that the increase of piezoresponse is principally due to the reduced strain and substrate clamping, as well as the reduced $a$-domains in 
nanostructured ferroelectrics. The sharp decrease in the piezoresponse of islands with a thickness below $20 \mathrm{~nm}$ is probably due to the increase in the relative volume of distorted regions affected by defects such as misfit dislocations [24].

By the structural analysis and electrical measurements, the abundance of $a$-domains and the piezoresponse were investigated as a function of the size of the ferroelectric nanostructures. However, the sizes of the ferroelectric nanostructures and their standard deviations were too wide to be accurate and practical. Another fabrication method to achieve rather uniformly sized ferroelectric nanostructures is still required. In the next session, we introduce a novel method for fabrication of even smaller ferroelectric nanocapacitors by means of a stencil mask.

Domain structures and piezoelectric properties in ferroelectric nanocapacitors

In the previous sections, ferroelectric nanostructures with various sizes were prepared and relations between their domain structures and piezoelectric properties were discussed. The reduction of substrate clamping effects and strains lead to the presence of non-tilted $a$-domains and an increase of the piezoresponse in the nanostructures. In this section, we would like to report on a novel method for the fabrication of ferroelectric nanocapacitors and on the investigation of their domain structures.

By ultra-thin anodic alumina masks (UTAAM), 64-nm sized $\mathrm{Pt} / \mathrm{Pb}\left(\mathrm{Zr}_{0.2} \mathrm{Ti}_{0.8}\right) \mathrm{O}_{3} / \mathrm{Pt}$ ferroelectric nanocapacitors were prepared. A two-step anodization process was employed to obtain well-defined masks with very small thickness. Figure 7 shows the evolution of current as a function of anodization time for (a) the first anodization and (b) the second anodization under the conditions of $0.3 \mathrm{M}$ of oxalic acid at $40 \mathrm{~V}$, at room temperature. Figure $8 \mathrm{a}$ shows a scheme of the deposition procedure. The AAO mask was transferred to the substrate of choice, and then the $\mathrm{Pb}\left(\mathrm{Zr}_{0.2} \mathrm{Ti}_{0.8}\right) \mathrm{O}_{3}$ material was deposited, e.g., onto $\mathrm{Pt}(100) / \mathrm{MgO}$ (100) substrate by PLD. Figure $8 \mathrm{~b}$ and inset show representative SEM images of an ultra-thin AAO mask with a pore size of $30 \mathrm{~nm}$ synthesized by anodization in sulfuric acid. The AAO mask is placed on the substrate. The inset shows a magnified image. Deposition was carried out at high temperature, for example, at $650{ }^{\circ} \mathrm{C}$, which makes it possible to enhance the mobility of the adsorbed particles on the surface of the mask avoiding the closure of the pore mouth and also to grow epitaxial $\mathrm{Pb}\left(\mathrm{Zr}_{0.2} \mathrm{Ti}_{0.8}\right) \mathrm{O}_{3}$ nanoislands directly, without post-annealing. The deposition pressure was below $10^{-6}$ Torr. The high-temperature deposition and the excellent stability of the AAO mask enabled us to deposit platinum for top electrodes by sputtering through the holes again. The SEM image in Fig. $8 \mathrm{c}$ shows the Pt/PZT/Pt nanocapacitors with size of $64 \mathrm{~nm}$ and interpore distance of $104 \mathrm{~nm}$, which is equivalent to a potential memory density of $68 \mathrm{~Gb} / \mathrm{inch}^{2}$. The mask was partly removed after the deposition process to visualize the nanoislands remaining on the substrate. The pore mouths were still open even after the PLD and Pt sputtering due to the highly mobilized particles at growth temperature, which means that it is possible to realize multilayered nanostructures using the AAO mask. Another advantage of the growth of $\mathrm{Pb}\left(\mathrm{Zr}_{0.2} \mathrm{Ti}_{0.8}\right) \mathrm{O}_{3}$ nanoislands at elevated growth temperature is the avoidance of deformation of the nanoislands during the post-crystallization process if the deposition would be performed at room temperature. Nanoislands deposited at room temperature were completely fragmentized by a high-temperature annealing treatment, although the deposition was carried out successfully and the mask preserved its original form (not shown here). Deformations of nanostructures by high-temperature treatment were reported before [33, 63], mainly due to densification of the amorphous islands deposited at room temperature resulting in shrinkage of the nanostructures during the crystallization process. In our case, the 64-nm sized nanoislands are too tiny to be stable under such harsh conditions. Therefore, the application of a high-temperature stable AAO mask is significant in this case.

Cross-section TEM images confirmed that the average height of the nanocapacitors was $40 \mathrm{~nm}$ as shown in
Fig. 7 Current flow as a function of anodization time for a the first anodization for $12 \mathrm{~h}$ and $\mathbf{b}$ the second anodization for 2 min using $\mathrm{H}_{2} \mathrm{C}_{2} \mathrm{O}_{4}$ electrolyte and $40 \mathrm{~V}$. The cartoon in a illustrates the formation procedure of porous AAO from a surface-finished aluminum plate
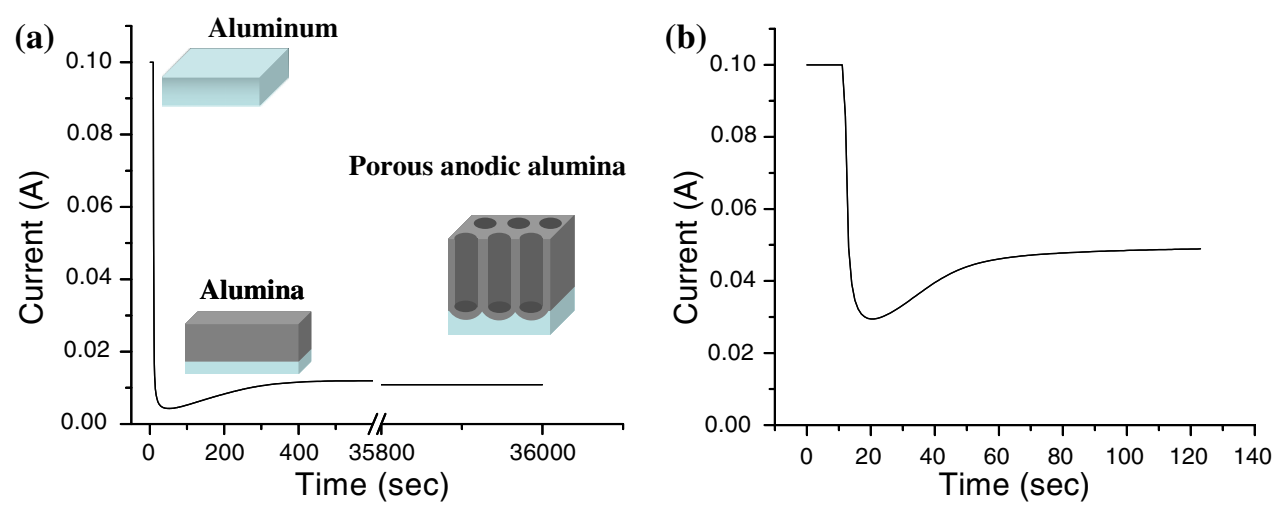
Fig. 8 a Schematics showing the PLD process of $\mathrm{Pb}\left(\mathrm{Zr}_{0.2} \mathrm{Ti}_{0.8}\right) \mathrm{O}_{3}$ material through the ultra-thin AAO mask onto a Pt bottom layer on $\mathrm{MgO}$ substrate, followed by $\mathrm{Pt}$ sputter deposition and lift-off of the mask leaving the PZT nanocapacitors on the substrate. b SEM images of the ultra-thin AAO mask and its magnified image. c PZT nanocapacitor arrays with remaining AAO mask to visualize the same ordering state of the PZT capacitors (From Ref. [58]) (a)

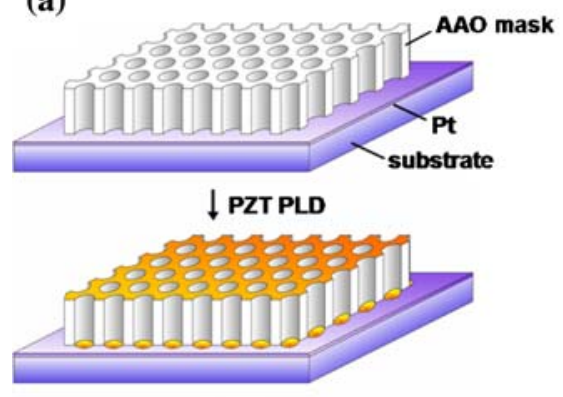

$\downarrow$ Pt PLD

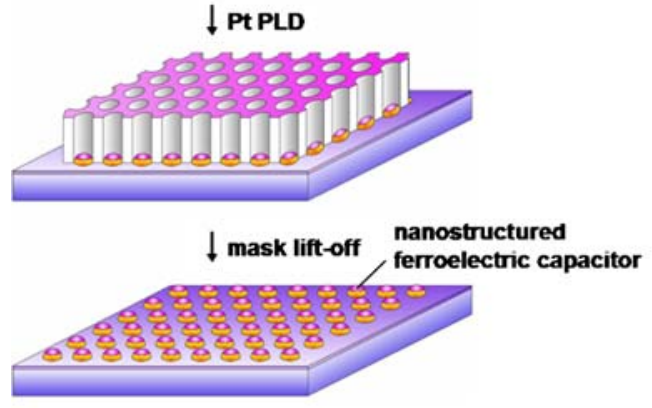

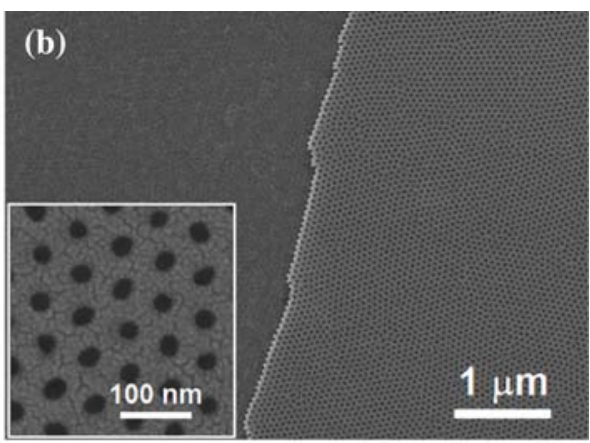

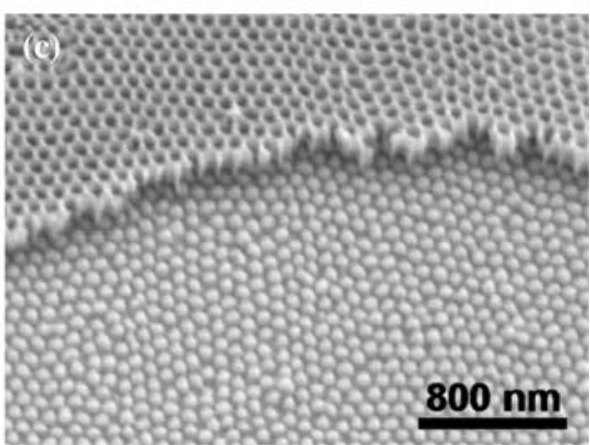

Fig. 9a. High-resolution TEM images proved that the nanocapacitors have a dome-like shape and a singlecrystalline quality (Fig. 9b). An entire Pt/PZT/Pt capacitor is shown in Fig. 9c. Figure 9d and e show the electron diffraction analysis indicating the epitaxial nature of both the Pt bottom electrode and the PZT nanoislands. Extended arrays of PZT nanocapacitors with high quality made it possible to investigate the structure and properties indepth. It was confirmed by $\theta-2 \theta$ and in-plane $\varphi$-scans that the nanocapacitors were grown epitaxially. The nanocapacitors preserved a high tetragonality of 1.048. The domain structures within the nanocapacitors were characterized by reciprocal space mapping near $\mathrm{Pb}\left(\mathrm{Zr}_{0.2} \mathrm{Ti}_{0.8}\right) \mathrm{O}_{3}$ (001) and (100) peaks using high-resolution synchrotron XRD as shown in Fig. 10. Three domain configurations, viz. a major fraction of $c$-domains (90\%), 4-fold symmetric $a$-domains (7\%) and symmetric $a$-domains (3\%) of minor fractions corresponding to PZT (001) and PZT (100), respectively, are present in the XRD contour map of the $H L$-plane. The symmetric $a$-domain present in the center of the 4-fold symmetric $a$-domains with the same value of the reciprocal lattice coordinate $h$ denotes that it has the same lattice parameter along the substrate normal direction and is exactly aligned parallel to the plane of the underlying bottom layers with a tilting angle of $\Delta \omega=0$. As mentioned in the previous section, this type of $a$-domains, i.e., non-tilted $a$-domains, can be attributed to a high degree of misfit strain relaxation in nanostructured ferroelectrics [4, 62, H. Han et al., unpublished]. The nontiled $a$-domains also were observed in $\mathrm{Pb}\left(\mathrm{Zr}_{0.2} \mathrm{Ti}_{0.8}\right) \mathrm{O}_{3}$ nanoislands epitaxially grown on $\mathrm{Nb}$-doped $\mathrm{SrTiO}_{3}$.
However, no twinned $a$-domains were detected in the STO case at all.

In order to investigate the electrical properties and contribution of domains to the piezoresponse, PFM measurements were carried out using conductive AFM tips. Figure 11 shows (a) the AFM topography image and (b) the PFM piezoresponse image of PZT nanoislands without Pt top electrodes. The as-grown PZT nanoislands contain $a$-domains as shown by horizontal lines in the $c$-domain matrix in the piezoresponse image, confirming the observations by XRD reciprocal space mapping. Effects of the high degree of misfit strain relaxation and the presence of $a$-domains in the PZT nanocapacitors were revealed by local hysteresis measurements. The latter were performed on individual PZT nanocapacitors. The piezoelectric constant $d_{33}$ of these PZT nanocapacitors was about $100 \mathrm{pm} / \mathrm{V}$, comparable to the theoretical value, while that of PZT patterns with a size of $20 \mu \mathrm{m}$, which can substitute for continuous ferroelectric structures, was about $70 \mathrm{pm} / \mathrm{V}$ as shown in Fig. 12a. The increase of the piezoelectric constant due to the high degree of strain relaxation and $a$-domain switching by an electric field in $1 \mu \mathrm{m}$ size PZT patterns has already been reported by Nagarajan [28]. Our 64-nm sized PZT nanocapacitors also showed an increase of piezoelectric properties due to the reduced misfit strain. Moreover, $a$-domain switching occurred in the PZT nanocapacitors as shown in Fig. 12b. By sweeping the voltage from -5 to $5 \mathrm{~V}$ first, a piezoelectric constant of about $120 \mathrm{pm} / \mathrm{V}$ was obtained, and then it decreased to $100 \mathrm{pm} / \mathrm{V}$ during the second voltage sweeping. Most probably, the $a$-domains were switched during the first hysteresis run 



(c)

)

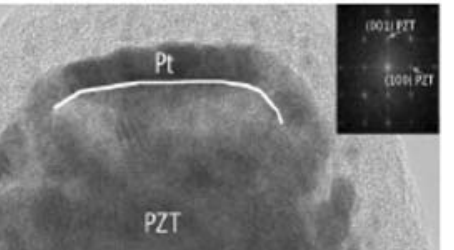

Fig. 9 a Cross-sectional TEM image of PZT nanoislands grown on $\mathrm{Pt} / \mathrm{MgO}$ (001). b High-resolution TEM image of a single PZT nanoisland on $\mathrm{Pt} / \mathrm{MgO}$ (001). c Cross-sectional TEM image of

Pt/PZT/Pt nanocapacitors. d, e Electron diffraction patterns obtained at the interfaces of $\mathrm{Pt} / \mathrm{MgO}(001)$ and $\mathrm{PZT} / \mathrm{Pt}(001)$, respectively (From Ref. [58])

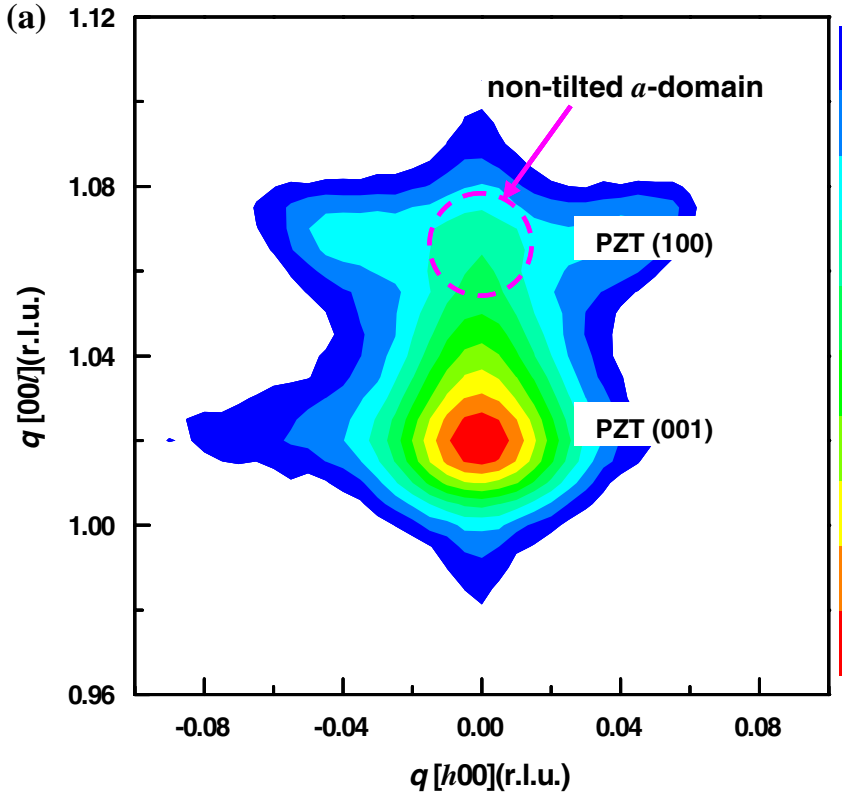

Fig. 10 Contour plots of reciprocal space mapping of $\mathrm{Pb}\left(\mathrm{Zr}_{0.2} \mathrm{Ti}_{0.8}\right) \mathrm{O}_{3}$ nanoislands. a $H L$-plane scan of $\mathrm{Pb}\left(\mathrm{Zr}_{0.2} \mathrm{Ti}_{0.8}\right) \mathrm{O}_{3}$ (001) and (100) reflections, b $H K$-plane scan of (100) reflection, and
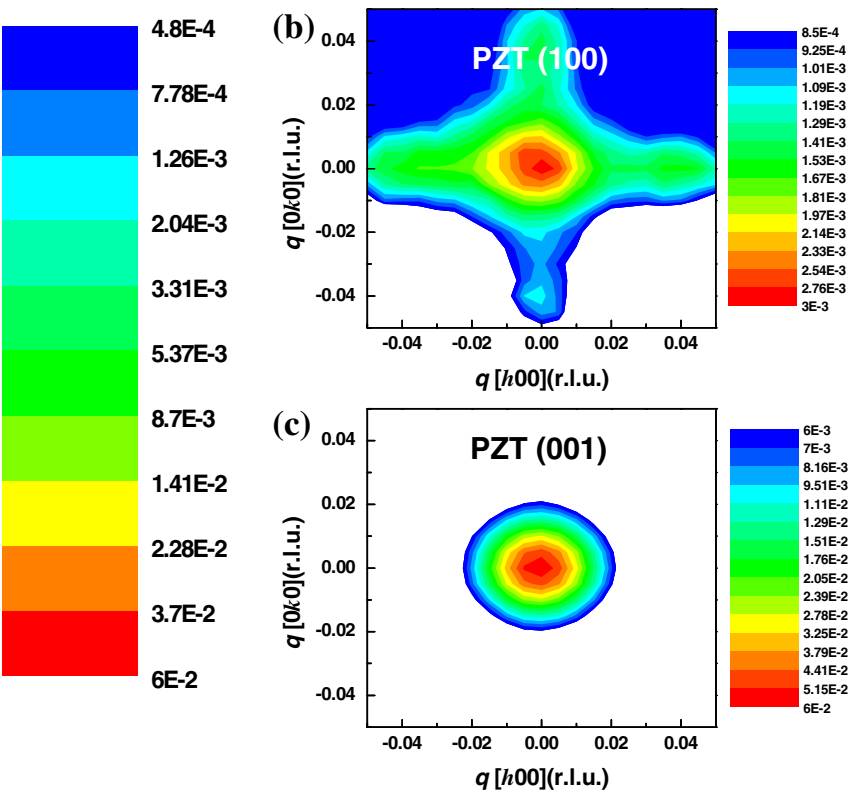

c $H K$-plane scan of (100) reflection corresponding to $a$-domain and $c$ domain, respectively (From Ref. [58]) 
Fig. 11 PFM measurement. a Topography and $\mathbf{b}$ piezoresponse images of $\mathrm{Pb}\left(\mathrm{Zr}_{0.2} \mathrm{Ti}_{0.8}\right) \mathrm{O}_{3}$ nanoislands with a size of $64 \mathrm{~nm}$. $a$-domain structures are visible in the piezoresponse image (From Ref. [58]) (a)

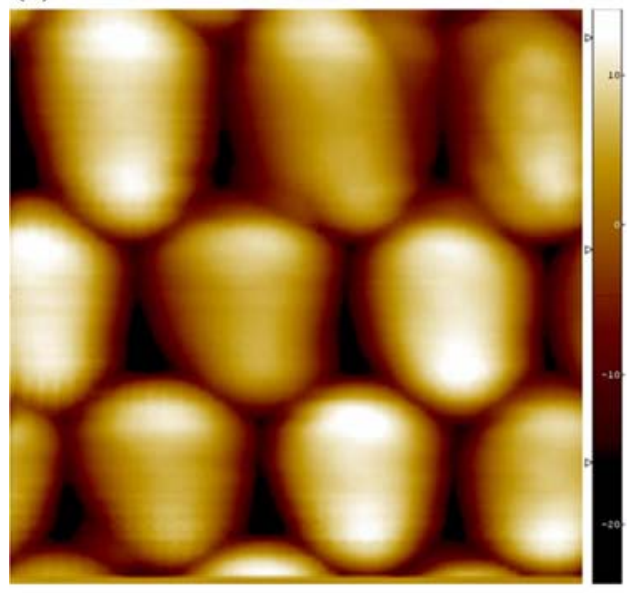

(b)

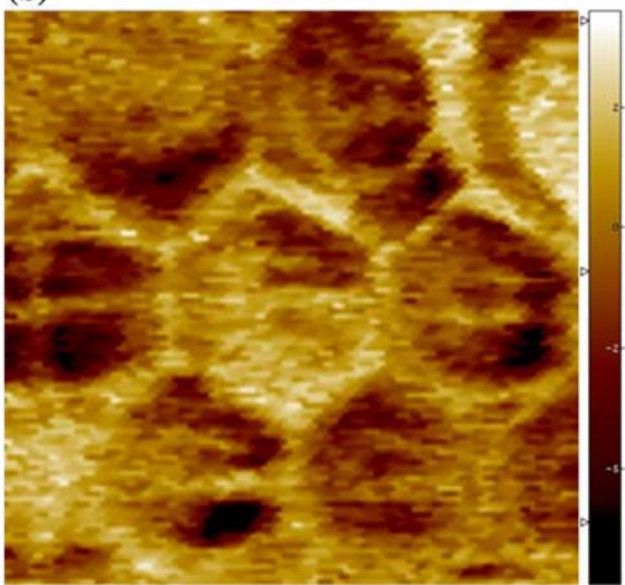

Fig. 12 Local piezoelectric hysteresis loops obtained from a $\mathrm{Pb}\left(\mathrm{Zr}_{0.2} \mathrm{Ti}_{0.8}\right) \mathrm{O}_{3}$ nanocapacitors (square) and $20-\mu \mathrm{m}$ sized pattern (circle) b Hysteresis loops obtained from $\mathrm{Pb}\left(\mathrm{Zr}_{0.2} \mathrm{Ti}_{0.8}\right) \mathrm{O}_{3}$ nanocapacitors by 1 st hysteresis run (circle) and 2nd hysteresis run (square) (From Ref. [58])

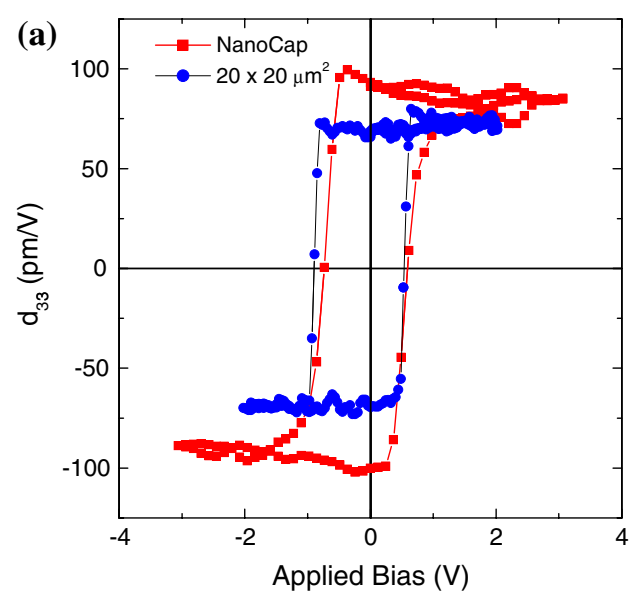

$100 \mathrm{~nm}$

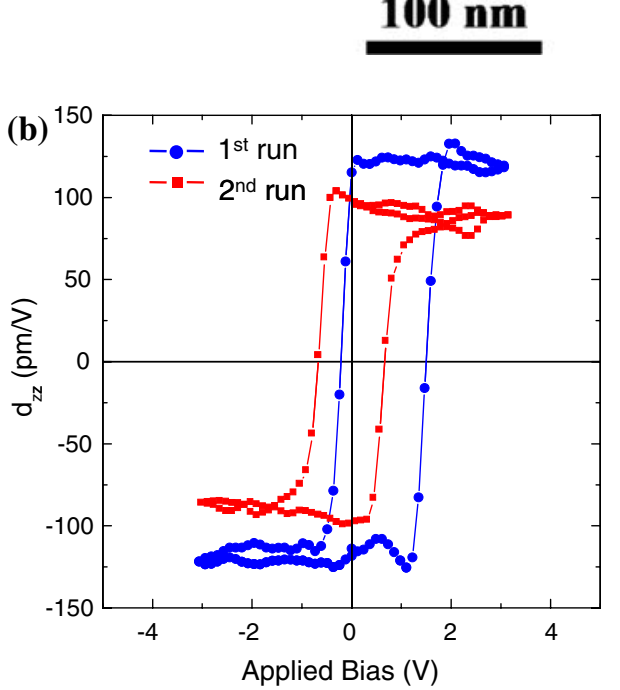

resulting in an increase of the piezoelectric constant, and then the value returned to its original value due to the elimination of switchable $a$-domains during the second hysteresis run. We are not sure whether the non-tilted $a$-domains were switched under the electric field at this moment. However, as Jesse et al. [55] reported, $a$-domain boundaries can serve as nucleation sites for $180^{\circ}$ switching and generally facilitate good switching properties.

Ferroelectric nanodisks and nanorings

There are still requirements for large area arrayed various ferroelectric nanostructures with narrow size distribution for applying them to practical devices. By LIL we were able to obtain wafer-scale arrays of $\mathrm{Pb}\left(\mathrm{Zr}_{0.2} \mathrm{Ti}_{0.8}\right) \mathrm{O}_{3}$ nanodisks and nanorings epitaxially grown on $\mathrm{SrRuO}_{3}$ $(001) / \mathrm{SrTiO}_{3}$ (001) [63]. The fabrication of ferroelectric nanodisks and nanorings consists of two steps, viz. (i) deposition at room temperature and (ii) post-annealing for crystallization. PLD was performed in vacuum, for example at a background pressure of $10^{-6}$ Torr in order to provide high energy to the PZT particles so that they can reach the bottom of the holes. Post-annealing was carried out at $650-700{ }^{\circ} \mathrm{C}$ for $1 \mathrm{~h}$ in $\mathrm{PbO}$ atmosphere to compensate for losses of volatile lead. The amount of amorphous PZT deposited into the patterned holes and its crystallization temperature determine the shape and size of the final PZT structures. Figure 13 shows $350 \mathrm{~nm}$ sized (a) $\mathrm{SiO}_{2}$ hole patterns, (b) $\mathrm{Pb}\left(\mathrm{Zr}_{0.2} \mathrm{Ti}_{0.8}\right) \mathrm{O}_{3}$ nanodisks, and (c) $\mathrm{Pb}\left(\mathrm{Zr}_{0.2} \mathrm{Ti}_{0.8}\right) \mathrm{O}_{3}$ nanorings fabricated by the two step process, viz. deposition at room temperature and crystallization. The crystallization into the ferroelectric phase nucleates at the edge regions near the free surfaces as well as at the interfaces of the nanodisks, and propagates gradually to the central part. The densification of the amorphous PZT with crystallization proceeds by a migration of some amorphous material to the crystallized ferroelectric during post-annealing, promoted by a high thermal energy as shown in the TEM image in Fig. 14. Accordingly, extended deposition enabled us to obtain ferroelectric nanodisks while nanorings could be obtained by only the condition of short deposition and high annealing 


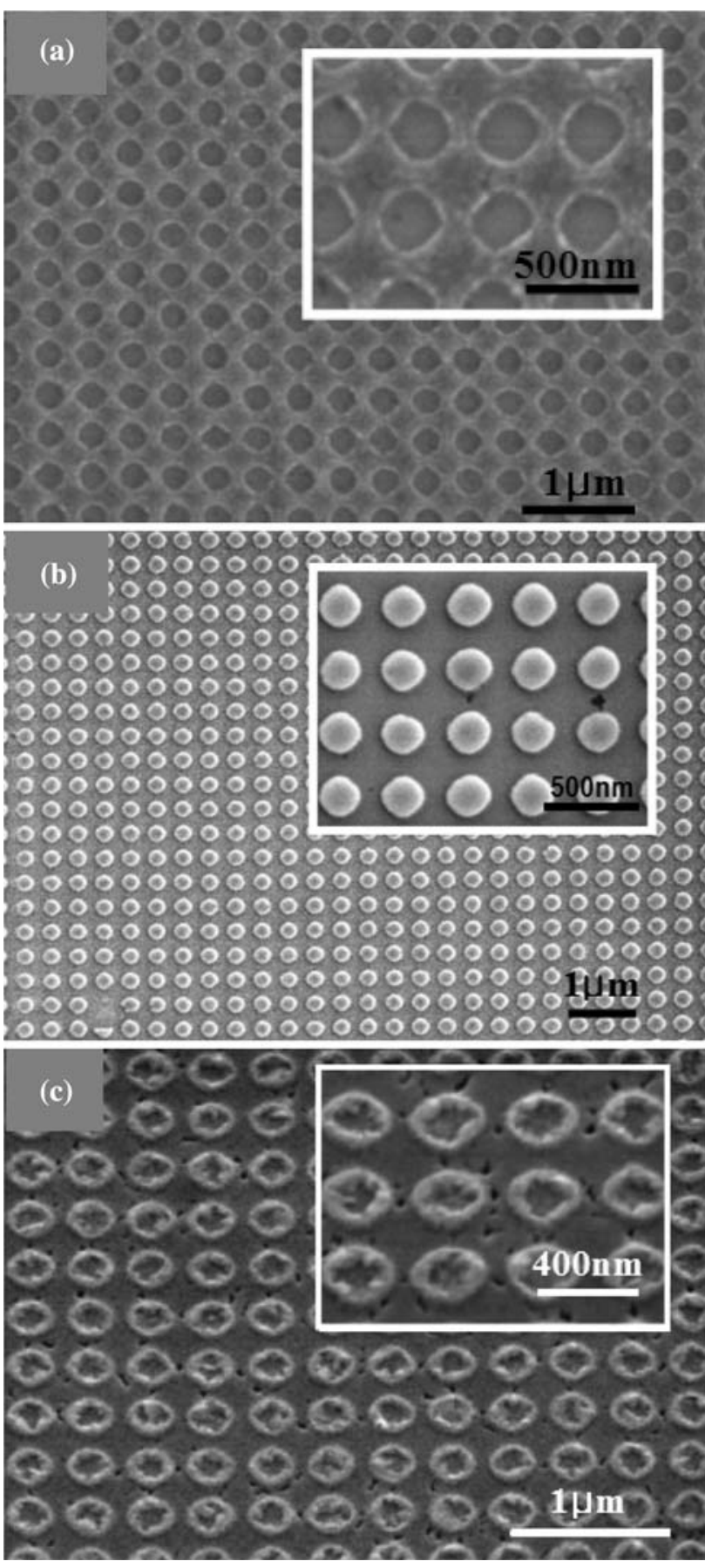

Fig. 13 Representative $\mathrm{SEM}$ images of a patterned $\mathrm{SiO}_{2}$ mask by LIL combined with RIE process, $\mathbf{b} \mathrm{Pb}\left(\mathrm{Zr}_{0.2} \mathrm{Ti}_{0.8}\right) \mathrm{O}_{3}$ nanodisks, and $\mathbf{c}$ $\mathrm{Pb}\left(\mathrm{Zr}_{0.2} \mathrm{Ti}_{0.8}\right) \mathrm{O}_{3}$ nanorings with size of $350 \mathrm{~nm}$ and large area uniform arrays (From Ref. [63])

temperature $\left(700{ }^{\circ} \mathrm{C}\right)$. Crystallized nanodisks and nanorings proved to be ferroelectric and showed a stable switching behavior under external field by PFM. Nanodisks and nanorings are interesting structures because a toroidal ordering of polarization was predicted to occur in those structures with a diameter of a few nanometers [31]. Zhu et al. [50] have achieved an internal diameter of $5 \mathrm{~nm}$ in

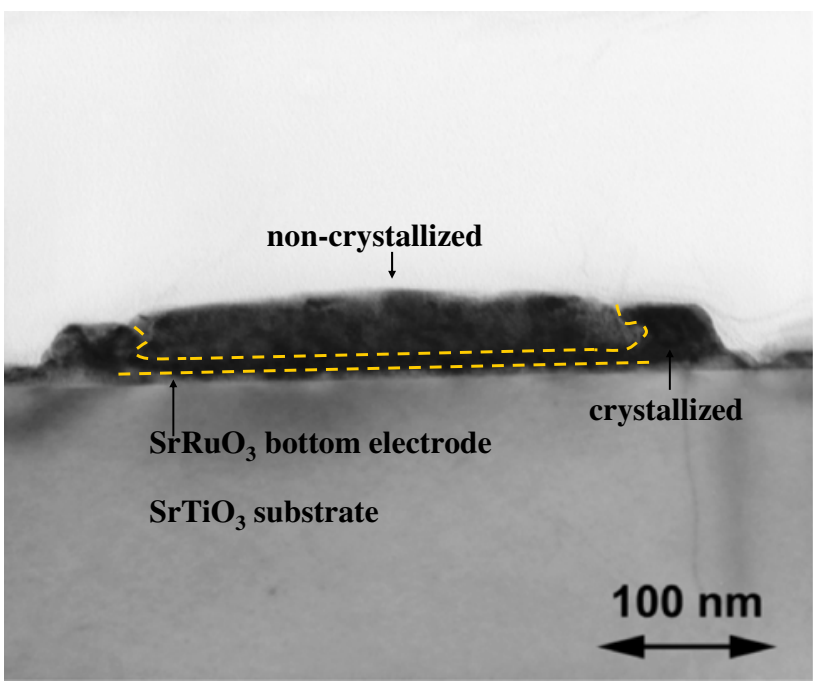

Fig. 14 Cross-sectional TEM image of $\mathrm{Pb}\left(\mathrm{Zr}_{0.2} \mathrm{Ti}_{0.8}\right) \mathrm{O}_{3}$ nanodisks grown on $\mathrm{SrRuO}_{3}$ bottom electrode and $\mathrm{SrTiO}_{3}$ substrate. By extended deposition and $650{ }^{\circ} \mathrm{C}$ annealing, edge and bottom of the nanodisks were crystallized and densified (guided with dashed line) (From Ref. [63])

PZT nanorings using an anodic alumina template and a conformal solution deposition through it. The LIL process combined with PLD so far provides rather large ferroelectric nanodisks and nanorings. However, as soon as laser sources with shorter wavelength become available for the LIL process, one will be able to achieve smaller sized ferroelectric nanodisks and nanorings on wafer-scale sizes.

\section{Conclusions}

In order to investigate the size effect and the domain structures in ferroelectric nanostructures, we fabricated $\mathrm{PbTiO}_{3}$ and $\mathrm{Pb}\left(\mathrm{Zr}_{0.2} \mathrm{Ti}_{0.8}\right) \mathrm{O}_{3}$ nanoislands and nanocapacitors employing a top-down method (e-beam lithography), a bottom-up technique (chemical solution deposition), and selective growth through stencils. The latter were obtained by LIL and by an ultra-thin anodic alumina process. The 100nm sized $\mathrm{PbTiO}_{3}$ islands were achieved by e-beam lithography for X-ray diffraction analysis. The CSD method enabled us to grow epitaxial $\mathrm{PbTiO}_{3}$ nanoislands on $\mathrm{Pt}(001) /$ $\mathrm{MgO}(001)$ and vary the size of ferroelectric nanoislands between 450 and $55 \mathrm{~nm}$ by controlling the dilution of the solutions. Using AAO masks and pulsed-laser deposition, we were able to synthesize 64-nm sized ferroelectric nanoislands and multilayered $\mathrm{Pt} / \mathrm{PZT} / \mathrm{Pt}$ nanocapacitors on $\mathrm{MgO}$ (001) and $\mathrm{Nb}$-doped $\mathrm{SrTiO}_{3}$ substrates, taking advantage of the good thermal stability of this ceramic stencil material. LIL is a most useful approach to obtain uniform wafer-scale arrays of ferroelectric nanodisks and nanorings by controlling the experimental conditions. The domain structure of all 
these ferroelectric nanostructures was characterized by reciprocal space mapping using high-resolution synchrotron $\mathrm{XRD}$ and AFM in the piezoresponse mode. When the size of the nanostructured ferroelectrics decreases down to $100 \mathrm{~nm}$ with scaling ratio of 1 , a new type of $a$-domains with a tilting angle $\Delta \omega=0$ was observed and thus periodic $c / a / c / a$ polydomain structures became unstable. This appearance of non-tilted $a$-domains is a clear evidence of misfit strain relaxation in the ferroelectric nanostructures. The piezoelectric properties could be enhanced by the reduced $a$-domains and substrate clamping effects in nanostructured ferroelectrics. PZT nanocapacitors with 64-nm size showed three domain configurations of $c$-domains, tilted $a$-domains, and non-tilted $a$-domains. PFM measurements confirmed that the piezoelectric constant of the nanocapacitors was higher than that of a ferroelectric continuous medium. $a$-domains could be switched under an external electric field due to the reduced misfit strain in nanostructured ferroelectrics. The twinned $90^{\circ}$ domain boundaries observed in PFM images also can play a role as nucleation sites of $180^{\circ}$ domain switching and can be a possible reason for the increase of the piezoelectric properties in PZT nanocapacitors. The questions "how small can nanostructured ferroelectrics become while still preserving ferroelectricity" and "will a singledomain state be stable in such nanostructures" have still not yet been answered conclusively. To investigate these questions, further new approaches for the fabrication of even smaller-sized ferroelectrics will be required.

Acknowledgements The authors thank Y. J. Park, J.-Y. Choi (Pohang Light Source, Pohang, Korea), S. Lee, Y. H. Jeong (Pohang University of Science and Technology, Pohang, Korea), R. Hillebrand, R. Ji, S. K. Lee, A. Lotnyk, M. A. Schubert, S. Senz, and U. Gösele (Max Planck Institute of Microstructure Physics, Halle, Germany), as well as K. Nielsch (Institute of Applied Physics, Hamburg, Germany) for many fruitful discussions and for experimental and analytical contributions. H. Han is grateful for the award of a fellowship of the German Academic Exchange Service (DAAD) and for support by DFG (446 KOR 113/215/0-1) and KRF. Financial support from the Volkswagen Foundation (Project I/80897), the German Ministry of Education and Research (BMBF, FKZ 03N8701) and from the Brain Korea 21 Project are also acknowledged. The experiments at PLS were supported by the MEST (Ministry of Education, Science, and Technology) and POSTECH.

Open Access This article is distributed under the terms of the Creative Commons Attribution Noncommercial License which permits any noncommercial use, distribution, and reproduction in any medium, provided the original author(s) and source are credited.

\section{References}

1. Scott JF, de Araujo CA (1989) Science 246:1400

2. Auciello O, Scott JF, Ramesh R (1998) Phys Today 51:22

3. Scott JF (2000) Ferroelectric memories. Springer-Verlag, New York
4. Lee K, Baik S (2006) Annu Rev Mater Sci 36:81

5. Shaw TM, Trolier-McKinstry S, McIntyre PC (2000) Annu Rev Mater Sci 30:263

6. Li S, Eastman JA, Li Z, Foster CM, Newnham RE, Cross LE (1996) Phys Lett A 212:341

7. Ahn CH, Rabe KM, Triscone J-M (2004) Science 303:488

8. Waser R, Rüdiger A (2004) Nat Mater 3:81

9. Fong DD, Sephenson GB, Streiffer SK, Eastman JA, Auciello O, Fuoss PH, Thompson C (2004) Science 304:1650

10. Nagrajan V, Junquera J, He JQ, Jia CL, Waser R, Lee K, Kim YK, Baik S, Zhao T, Ramesh R, Gosez Ph, Rabe KM (2006) J Appl Phys 100:051609

11. Tybell T, Ahn CH, Triscone J-M (1999) Appl Phys Lett 75:856

12. Lichtensteiger C, Trinsone J-M (2005) Phys Rev Lett 94: 047603

13. Streiffer SK, Eastman JA, Fong DD, Thompson C, Munkholm A, Ramana Murty MV, Auciello O, Bai GR, Stephenson GB (2002) Phys Rev Lett 89:067601-1

14. Kornev IA, Fu H, Bellaiche L (2006) J Mater Sci 41:137. doi: 10.1007/s10853-005-5962-0

15. Kim J, Yang SA, Choi YC, Han JK, Jeong KO, Yun YJ, Kim DA, Yang SM, Yoon D, Cheong H, Chang KS, Noh TW, Bu SD (2008) Nano Lett 8:1813

16. Luo Y, Szafraniak I, Zakharov ND, Nagarajan V, Steinhart M, Wehrspohn RB, Wendorff JH, Ramesh R, Alexe M (2003) Appl Phys Lett 83:440

17. Urban JJ, Spanier JE, Ouyang L, Yun WS, Park H (2003) Adv Mater 15:423

18. Gu H, Hu Y, You J, Hu Z, Yuan Y, Zhang T (2007) J Appl Phys 101:024319

19. Xu G, Ren ZH, Du PY, Weng WJ, Shen G, Han GR (2005) Adv Mater 17:907

20. Junquera J, Ghosez Ph (2003) Nature 422:506

21. Gruverman A, Kalinin SV (2006) J Mater Sci 41:107. doi:10.1007/ s10853-005-5946-0

22. Sepliarsky M, Stachiotti MG, Migoni RL (2006) Phys Rev Lett 96:137603

23. Nagarajan V, Jia CL, Kohlstedt H, Waser R, Misirlioglu IB, Alpay SP, Ramesh R (2005) Appl Phys Lett 86:192910

24. Chu M-W, Szafraniak I, Scholz R, Harnagea C, Hesse D, Alexe M, Gösele U (2004) Nat Mater 3:87

25. Wang YG, Zhong WL, Zhang PL (1995) Phys Rev B 51:5311

26. Wang YG, Zhong WL, Zhang PL (1995) Phys Rev B 51:17235

27. Kretschmer R, Binder K (1979) Phys Rev B 20:1065

28. Nagarajan V, Roytburd A, Stanishevsky A, Prasertchoung S, Zhao T, Chen L, Melngailis J, Auciello O, Ramesh R (2003) Nat Mater 2:43

29. Lee K, Baik S (2005) Appl Phys Lett 86:202901

30. Bühlmann S, Dwir B, Baborowski J, Muralt P (2002) Appl Phys Lett 80:3195

31. Kim YK, Kim SS, Shin H, Baik S (2004) Appl Phys Lett 84:5085

32. Naumov II, Bellalche L, Fu H (2004) Nature 432:737

33. Alexe M, Harnagea C, Hesse D, Gösele U (1997) Appl Phys Lett 75:1793

34. Alexe M, Harnagea C, Hesse D, Gösele U (2001) Appl Phys Lett 79:242

35. Ganpule CS, Stanishevsky A, Su Q, Aggarwal S, Melngailis J, Williams E, Ramesh R (1999) Appl Phys Lett 75:409

36. Nagarajan V, Stanishevsky A, Ramesh R (2006) Nanotechnology $17: 338$

37. Harnagea C, Alexe M, Schilling J, Choi J, Wehrspohn RB, Hesse D, Gösele U (2003) Appl Phys Lett 83:1827

38. Shimizu M, Nonomura N, Fujisawa H, Niu H, Honda K (2004) Integr Ferroelectr 62:109

39. Szafraniak I, Harnagea C, Scholz R, Bhattacharyya S, Hesse D, Alexe M (2003) Appl Phys Lett 83:2211 
40. Alexe M, Hesse D (2006) J Mater Sci 41:1. doi:10.1007/ s10853-005-5912-x

41. Roelofs A, Schneller T, Szot K, Waser R (2002) Appl Phys Lett 81:5231

42. Ahn SH, Choi SK (2008) Appl Phys Lett 93:113102

43. Alexe M, Harnagea C, Hesse D (2004) J Electroceramics 12:69

44. Fujisawa H, Okaniwa M, Nonomura H, Shimizu M, Niu H (2004) J Eur Ceram Soc 24:1641

45. Ma W, Hesse D, Gösele U (2005) Small 1:837

46. Lee W, Alexe M, Nielsch K, Gösele U (2005) Chem Mater $17: 3325$

47. Lee SK, Lee W, Alexe M, Nielsch K, Hesse D, Gösele U (2005) Appl Phys Lett 86:152906

48. Clemens S, Schneller T, van der Hart A, Peter F, Waser R (2005) Adv Mater 17:1357

49. Kronholz S, Rathgeber S, Karthäuser S, Kohlstedt H, Clemens S, Schneller T (2006) Adv Funct Mater 16:2346

50. Zhu XH, Evans PR, Byrne D, Schilling A, Douglas C, Pollard RJ, Bowman RM, Gregg JM, Morrison FD, Scott JF (2006) Appl Phys Lett 89:122913

51. Evans PR, Zhu X, Baxter P, McMillen M, McPhillips J, Morrison FD, Scott JF, Pollard RJ, Bowman RM, Gregg JM (2007) Nano Lett 7:1134
52. Damjanovic D (1998) Rep Prog Phys 61:1267

53. Ramesh R, Sands T, Keramidas VG (1993) Appl Phys Lett 63:731

54. Lee KS, Choi JH, Lee JY, Baik S (2001) J Appl Phys 90:4095

55. Jesse S, Rodriguez BJ, Choudhury S, Baddorf A, Vrejoiu I, Hesse D, Alexe M, Eliseev E, Morozovska AN, Zhang J, Chen L-Q, Kalinin SV (2008) Nat Mater 7:209

56. Jia C-L, Mi S-B, Urban K, Vrejoiu I, Alexe M, Hesse D (2008) Nat Mater 7:57

57. Masuda H, Fukuda K (1995) Science 268:1466

58. Lee W, Han H, Lotnyk A, Schubert MA, Senz S, Alexe M, Hesse D, Baik S, Gösele U (2008) Nat Nanotechnol 3:402

59. Speck JS, Pompe W (1994) J Appl Phys 76:466

60. Alpay SP, Roytburd AL (1998) J Appl Phys 83:4714

61. Lee K, Kim K, Kwon S-J, Baik S (2004) Appl Phys Lett 85:4711

62. Lee K, Yi H, Park W-H, Kim YK, Baik S (2006) J Appl Phys 100:051615

63. Han H, Park YJ, Lee SK, Ji R, Le Rhun G, Alexe M, Nielsch K, Hesse D, Gösele U, Baik S (2009) Nanotechnology 20:015301 